\title{
The Costs and Health Benefits of Expanded Access to MDMA-assisted Therapy for Chronic and Severe PTSD in the USA: A Modeling Study
}

\author{
Anton L. V. Avanceña ${ }^{1,2}$ (D) . James G. Kahn ${ }^{2,3,4,5} \cdot$ Elliot Marseille $^{2,6} \mathbb{D}$
}

Accepted: 26 January 2022 / Published online: 1 March 2022

(c) The Author(s) 2022

\begin{abstract}
Background and Objective Intensive psychotherapy assisted with 3,4-methylenedioxymethamphetamine (MDMA-AT) was shown in Phase 3 clinical trials to substantially reduce post-traumatic stress disorder (PTSD) symptoms compared to psychotherapy with placebo. This study estimates potential costs, health benefits, and net savings of expanding access to MDMA-AT to eligible US patients with chronic and severe PTSD.

Methods Using a decision-analytic model, we compared the costs, deaths averted, and quality-adjusted life years (QALYs) gained of three, 10-year MDMA-AT coverage targets $(25 \%, 50 \%$, and $75 \%)$ compared to providing standard of care to the same number of eligible patients with chronic and severe PTSD. We used a payer perspective and discounted costs (in US\$) and QALYs to 2020. We conducted one-way, scenario, and probabilistic sensitivity analyses and calculated the net monetary value of MDMA-AT using a cost-effectiveness threshold of $\$ 100,000$ per QALY gained.

Results Expanding access to MDMA-AT to $25-75 \%$ of eligible patients is projected to avert 43,618-106,932 deaths and gain 3.3-8.2 million QALYs. All three treatment targets are dominant or cost-saving compared to standard of care. Our sensitivity analyses found that accounting for parameter uncertainty and changes in various assumptions did not alter the main finding-MDMA-AT is dominant compared to standard of care.

Conclusion Expanding access to MDMA-AT to patients with chronic and severe PTSD will provide substantial health and financial benefits. The precise magnitude is uncertain and will depend on the number of eligible patients and other inputs.
\end{abstract}

Elliot Marseille

emarseille1@ berkeley.edu

Anton L. V. Avanceña

antonlv@umich.edu

James G. Kahn

jgkahn@gmail.com

1 Department of Health Management and Policy, University of Michigan School of Public Health, Ann Arbor, MI, USA

2 Global Initiative for Psychedelic Science Economics (GIPSE), UC Berkeley/UC San Francisco Center for Global Health Delivery, Diplomacy, and Economics, Berkeley, CA, USA

3 Philip R. Lee Institute for Health Policy Studies, School of Medicine, UC San Francisco, San Francisco, CA, USA

4 Department of Epidemiology and Biostatistics, School of Medicine, UC San Francisco, San Francisco, CA, USA

5 Institute for Global Health Sciences, UC San Francisco, San Francisco, CA, USA

6 Health Strategies International, 555 59th Street, Oakland, CA 94609, USA

\section{Key Points}

MDMA-AT has been shown in Phase 3 clinical trials to substantially reduce PTSD symptoms compared to psychotherapy with placebo.

Increasing access to MDMA-AT to $25-75 \%$ of eligible patients with chronic and severe PTSD over 10 years would avert between 43,000 and 106,000 deaths, produce 3.3-8.2 million discounted quality-adjusted life years, and lead to \$109-\$266 billions in discounted net savings for the healthcare system.

Increased access to MDMA-AT can save lives and improve the health of patients with chronic and severe PTSD, while reducing healthcare costs. 


\section{Introduction}

Post-traumatic stress disorder (PTSD) is a highly debilitating psychiatric condition that adversely affects mood, cognition, physical and emotional health, and can lead to suicidal ideation and behavior [1-4]. In any given year, 3.5-4.7\% of US adults or about 7.3-9.8 million individuals experience PTSD. Among military veterans, current prevalence of PTSD is as high as $13 \%$, although some cohorts of combat veterans have reported PTSD prevalence as high as $20 \%$ [5-8]. PTSD is associated with healthcare costs and service utilization that are higher than those of other mental health conditions [9-15].

While about half of people with PTSD achieve recovery or remission spontaneously or following standard of care (SoC) [16-18], the remainder suffer from chronic and severe forms of PTSD which require long-term treatment. Existing Food and Drug Administration (FDA)-approved pharmacotherapies, sertraline and paroxetine, however, have limited effectiveness and unwanted side effects [19-21]. Recently, a new treatment for PTSD involving 3,4-methylenedioxymethamphetamine (MDMA), a currently illegal psychedelic agent, combined with psychotherapy was shown in a multisite, Phase 3 randomized clinical trial to be significantly more effective in reducing PTSD symptoms than placebo with same course of psychotherapy in patients with severe or extreme PTSD. The trial also found that MDMA-assisted therapy (MDMA-AT) did not increase suicidal ideation and other adverse events, even among those with comorbidities such as dissociation and depression [22]. With such promising results, the FDA could approve MDMA-AT for PTSD by 2023 [23].

Previous cost-effectiveness analyses have shown that MDMA-AT is dominant, which means that it generates more health benefits and has lower net costs than SoC, even under conservative assumptions regarding the benefits of treatment [24, 25]. In this study, we aim to estimate the costs, health benefits, and net savings of increased access to MDMA-AT among US adult patients with chronic and severe PTSD.

\section{Methods}

\subsection{Overview}

Following the Consolidated Health Economic Evaluation Reporting Standards and guidelines from the 2nd Panel on Cost-effectiveness in Health and Medicine [26, 27], we developed a decision-analytic model that simulates a closed cohort of about 3.5 million adult patients (i.e., excluding incident cases) with chronic and severe PTSD who are eligible for MDMA-AT based on the inclusion and exclusion criteria used in the Phase 3 trial [22]. We used a payer perspective and discounted future costs and benefits using a $3 \%$ yearly rate over a 30-year time horizon in the base-case analysis. The model, which was programmed in Microsoft Excel ${ }^{\circledR}$ (Microsoft Corp, Redmond, WA), uses annual cycles.

We modeled three hypothetical MDMA-AT treatment targets: $25 \%, 50 \%$, and $75 \%$ (Fig. 1). These targets were selected because they are within the Healthy People 2030 goal for the proportion of adults with serious mental illness who receive treatment [28]. From year to year, the proportion of treated patients with chronic and severe PTSD nearly doubles until each cumulative target is reached at the end of 10 years. Our approach in constructing these treatment targets is based on previous modeling studies on the costs and benefits of scaling up mental health treatment services [29, 30].
Fig. 1 Cumulative proportion of eligible patients with chronic and severe PTSD who received with MDMA-AT over 10 years under three treatment targets. MDMA-AT 3,4-methylenedioxymethamphetamine assistedtherapy, $P T S D$ post-traumatic stress disorder

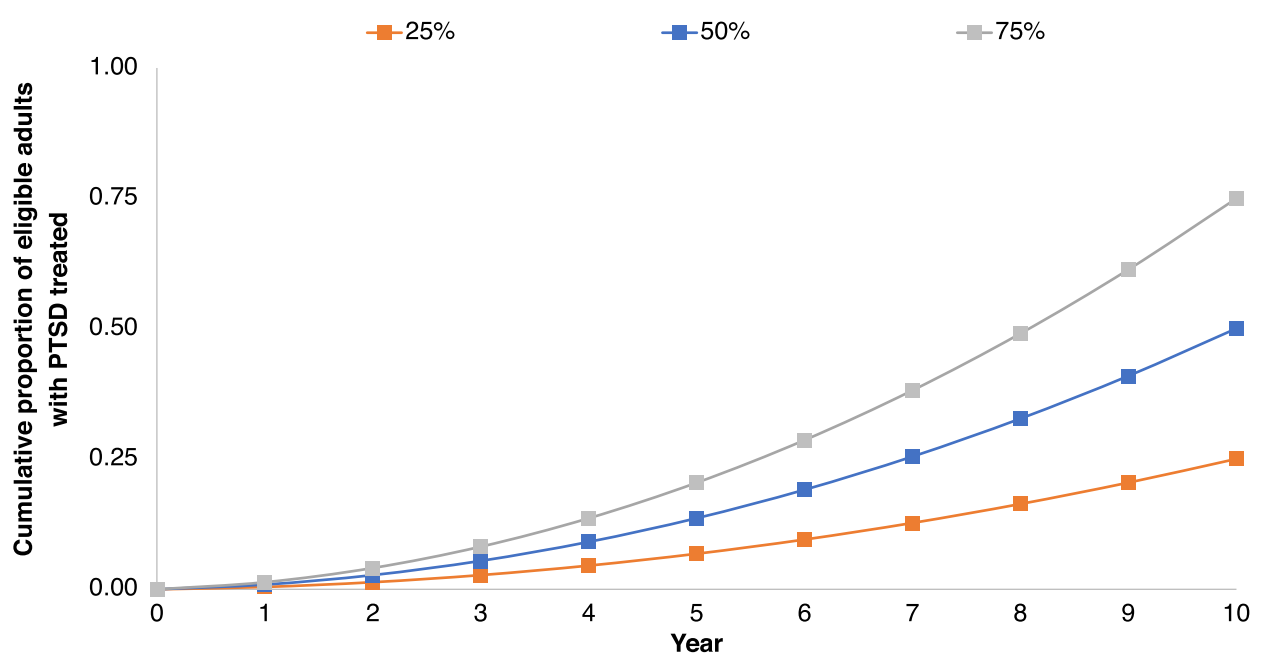




\subsection{Data Sources}

Data for the model (Table 1) were based on secondary sources. A full description of our estimation methods is presented in the Supplement.

\subsubsection{Patients Eligible for MDMA-AT}

We estimated the number of adult patients with chronic and severe PTSD who are eligible for MDMA-AT (Fig. 2) using published sources (see Electronic Supplementary Material

Table 1 Model inputs

\begin{tabular}{|c|c|c|c|}
\hline & Base value (range) & Distribution in PSA & Source \\
\hline \multicolumn{4}{|c|}{ Distribution of patients with PTSD who receive MDMA-AT by severity } \\
\hline Asymptomatic & 33.3 & NA & {$[24]$} \\
\hline Mild & 26.2 & NA & {$[24]$} \\
\hline Moderate & 26.2 & NA & [24] \\
\hline Severe & 11.9 & NA & {$[24]$} \\
\hline Extreme & 2.4 & NA & [24] \\
\hline Dead & 0 & NA & {$[24]$} \\
\hline \multicolumn{4}{|c|}{ Distribution of patients with PTSD who receive standard of care by severity } \\
\hline Asymptomatic & 0 & NA & [24] \\
\hline Mild & 0 & NA & [24] \\
\hline Moderate & 0 & NA & [24] \\
\hline Severe & 71.4 & NA & [24] \\
\hline Extreme & 28.3 & NA & [24] \\
\hline Dead & 0 & NA & [24] \\
\hline \multicolumn{4}{|l|}{ Costs (in 2020 US\$) ${ }^{b}$} \\
\hline MDMA-AT (one-time) & $11,537(8076-14,998)$ & Gamma & {$[55,56]^{\mathrm{a}}$} \\
\hline Standard of care & $0(0-13,256)$ & Gamma & {$[55,56]^{\mathrm{a}}$} \\
\hline Medical care for asymptomatic PTSD & $5032(3608-6456)$ & Gamma & {$[57-60]^{\mathrm{a}}$} \\
\hline Medical care for mild PTSD & $10,118(7250-12,986)$ & Gamma & {$[57-60]^{\mathrm{a}}$} \\
\hline Medical care for moderate PTSD & $15,177(10,855-19,469)$ & Gamma & {$[57-60]^{\mathrm{a}}$} \\
\hline Medical care for severe PTSD & $20,236(14,512-25,960)$ & Gamma & {$[57-60]^{\mathrm{a}}$} \\
\hline Medical care for extreme PTSD & $24,283(17,415-31,151)$ & Gamma & {$[57-60]^{\mathrm{a}}$} \\
\hline \multicolumn{4}{|l|}{ Health utilities } \\
\hline Asymptomatic & $0.90(0.81-0.99)$ & Beta & [24] \\
\hline Mild & $0.83(0.75-0.91)$ & Beta & [24] \\
\hline Moderate & $0.74(0.67-0.81)$ & Beta & {$[24]$} \\
\hline Severe & $0.61(0.55-0.67)$ & Beta & [24] \\
\hline Extreme & $0.37(0.33-0.41)$ & Beta & {$[24]$} \\
\hline Dead & 0 & NA & Assumed \\
\hline \multicolumn{4}{|l|}{ Other inputs and assumptions } \\
\hline Cohort size (range) & $3,523,049(2,110,743-4,735,640)$ & Normal & {$[16-18,31-33]^{\mathrm{a}}$} \\
\hline Annual discount rate $(\%)$ & $3(0.23-0.38)$ & Beta & Assumed \\
\hline Time horizon (years) & $30(10-40)$ & Uniform & Assumed \\
\hline Mean age of cohort & 41 & NA & [22] \\
\hline Probability of death from all causes & $0.002(0.0018-0.0022)$ & Beta & {$[61]$} \\
\hline
\end{tabular}

MDMA-AT 3,4-methylenedioxymethamphetamine assisted-therapy, NA not applicable, PSA probabilistic sensitivity analysis, PTSD post-traumatic stress disorder

${ }^{a}$ Base-case value and range calculated by authors using the references listed

${ }^{\mathrm{b}}$ Costs are annual unless otherwise noted 


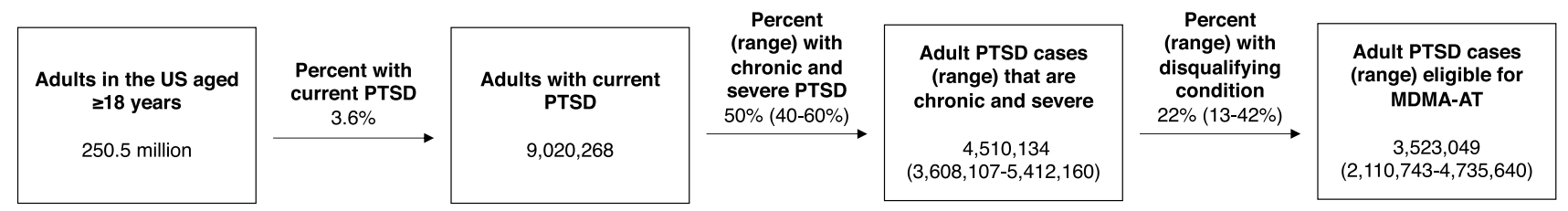

Fig. 2 Flow diagram summarizing the process for estimating the number of patients with chronic and severe PTSD who are eligible for MDMA-AT based on the eligibility criteria used in the Phase 3

[ESM]). Citing national survey data, the National Institute for Mental Health states that the past-year prevalence of PTSD among US adults is $3.6 \%$ [31]. Since there are $250,563,000$ people aged $\geq 18$ years in 2019 [32], we estimated that the number of adults with PTSD is 9,020,268.

Previous studies estimate that the proportion of people with PTSD who experience a chronic and severe form is $50 \%$ (range 40-60\%) [16-18]. Of this population, about $21.9 \%$ (range $12.5-41.5 \%$ ) would be ineligible for MDMA-AT because of psychiatric and medical comorbidities such as any current substance use disorder, primary psychotic disorder, and bipolar disorder according to the inclusion criteria of the Phase 3 trial [33]. Thus, in the base-case analysis we assumed that the number of eligible patients is 3,523,049 (range: 2,110,743-4,735,640).

\subsubsection{Transition Probabilities}

We based transition probabilities on the results of our previous modeling study [24]. We used a previously published trials of MDMA-AT. MDMA-AT methylenedioxymethamphetamineassisted psychotherapy, $P T S D$ post-traumatic stress disorder

state-transition model developed by two of the current authors that estimated the health and economic outcomes beyond the 8 -week time horizon of the Phase 3 trial (Fig. 3) [24]. In addition to deceased, the health states in the model were asymptomatic, mild, moderate, severe, and extreme PTSD, which are based on patient scores on the most recent iteration of the Clinician-Administered PTSD Scale.

Our prior study produced "Markov traces," or distributions of 1000 simulated patients across health states, for those who received MDMA-AT and SoC (Fig. S1-S2 and Tables S1-S2 in ESM). We used these traces to portray the proportion of simulated patients in each health state over time. For example, $71.4 \%$ of simulated patients who receive SoC start from the severe PTSD health state, while $28.6 \%$ begin from the extreme PTSD health state (Table 1). These patients progress to other health states based on the Markov trace for this population (Fig. S2 in ESM). Similarly, patients who receive MDMA-AT start from the asymptomatic $(33.3 \%)$, mild $(26.2 \%)$, moderate

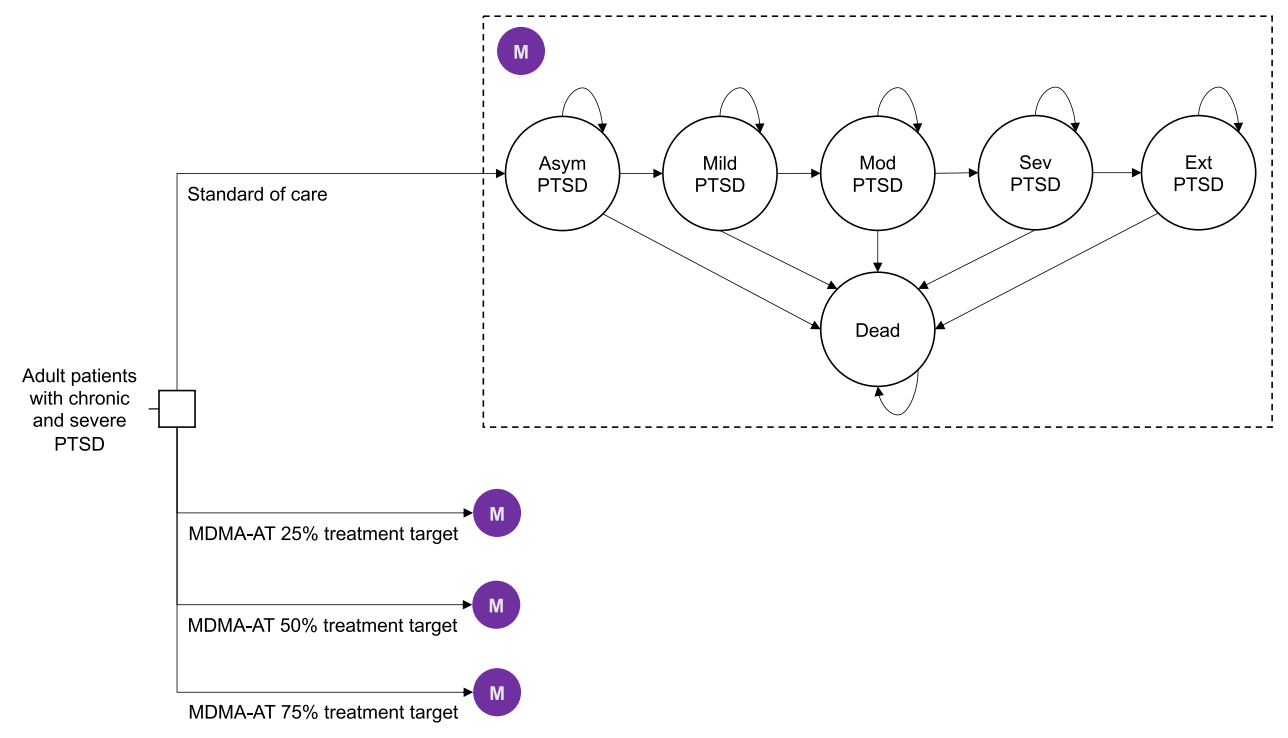

Fig. 3 Root of the schematic shows the four decision alternatives or scenarios-standard of care and the three MDMA-AT treatment targets $(25 \%, 50 \%$, and $75 \%$ of eligible patients). The shaded purple circle denotes the common Markov node, and the ovals are the PTSD states that simulated patients transition to. Each PTSD state is associ- ated with a cost and health utility. Arrows represent transitions and are associated with an annual probability. Asym asymptomatic, Ext extreme, MDMA-AT methylenedioxymethamphetamine-assisted psychotherapy, Mod moderate, PTSD post-traumatic stress disorder, Sev severe 
(28.6\%), severe $(9.5 \%)$, and extreme $(2.4 \%)$ health states (Table 1), and their trajectories follow the Markov trace for this population (Fig. S1 in ESM). The costs of treating PTSD, health state utilities, and annual mortality vary according to the severity of PTSD; thus, the results are determined by the proportion of patients in each severity category over time.

We assume that patients with PTSD are treated with the same MDMA-AT protocol as in the Phase 3 trial [19, 22, 34], and that they experience the same level of efficacy modeled in the prior study in the base-case analysis. While the long-term efficacy of MDMA-AT is still unknown, limited research suggests that remission from severe PTSD symptoms among patients who receive MDMA-AT was maintained for a follow-up period averaging 3.5 years following treatment [35]. In scenario analysis, we reduce the efficacy of MDMA-AT to understand the effect of this assumption on our results.

\subsubsection{Costs}

We included two main healthcare-related costs: the cost of MDMA-AT and the cost of medical care for patients with PTSD (Table 1). We briefly describe our methods for estimating these costs below, and full details are provided elsewhere [24]. All costs are in 2020 US\$.

The one-time cost of MDMA-AT $(\$ 11,537$, range: $\$ 8076-14,998$; Table 1) was based on a previous microcosting of the resources required to deliver MDMA-AT per protocol, including three 90-min preparatory psychotherapy sessions, three 8-h MDMA sessions, and nine 90-min integration sessions. All sessions are conducted with one patient and two clinicians.

The cost of medical care refers to all healthcare costs incurred by patients with PTSD in a year; these care costs are positively associated with PTSD severity according to previous research [36]. The costs of medical care for different PTSD severity levels were derived from estimates in the peer-reviewed literature (Table 1). In the base-case analysis, we assumed that the costs of SoC are included in medical care costs, although we varied this assumption in the sensitivity analyses.

\subsubsection{Health Outcomes}

The outcomes of interest in this study were deaths averted and quality-adjusted life years (QALYs) gained. QALYs combine length and quality of life and are the preferred health outcome in economic evaluations for health in developed countries [37]. QALYs in this study incorporate health utilities that were calculated using EQ-5D-5L scores of participants in the MDMA-AT Phase 3 trial (Table 1) [24]. Respondent scores on the EQ-5D-5L are converted to health utilities through a valuation method that uses preference weights specific to the USA [38].

\subsection{Analysis}

\subsubsection{Incremental Costs and Benefits}

For each treatment target, we calculated the incremental costs and health benefits of MDMA-AT versus SoC over 30 years (note: the time horizon extends beyond the 10-year scale-up period). The ratio of incremental costs to incremental health benefits, or the incremental cost-effectiveness ratio (ICER), is the net cost per unit of health outcome gained. The ICER is used to assess the cost effectiveness of an intervention by comparing it to a context-specific cost-effectiveness threshold. In this study, we used $\$ 100,000$ per QALY gained as the threshold, which is commonly used in the USA [37]. In instances where the incremental costs were negative, we did not report negative ICERs, which are uninterpretable.

\subsubsection{Sensitivity Analysis}

To generate base-case results for each treatment target, we used the base value of each input parameter (see Table 1) in the model. Then, to test the effect of parameter uncertainty and various assumptions on our results, we conducted oneway, scenario, and probabilistic sensitivity analyses (PSA).

In one-way sensitivity analysis, we varied each input parameter one at a time from their lowest to highest value (Table 1) to understand how extreme values affect the cost effectiveness of MDMA-AT. The results of one-way sensitivity analyses are presented in tornado diagrams that show how the net monetary value (NMV) of MDMA-AT changes as the value of an input changes. The NMV is a measure combining the costs and health benefits of an intervention into a single number and is calculated by multiplying the number of QALYs gained by a cost-effectiveness threshold (i.e., $\$ 100,000$ per QALY gained), and then subtracting from the product the total costs of the intervention. An intervention is deemed efficient if its NMV is positive, which means that the benefits of the intervention outweigh its costs.

In scenario analyses, we varied the effectiveness of MDMA-AT and SoC. In one scenario, we assumed that SoC is more effective and is associated with some reduction in PTSD severity (Fig. S3 in ESM). In a second scenario, we assumed that MDMA-AT's effectiveness declines over time, and that patients who receive MDMA-AT experience a $10 \%$ annual regression in their PTSD severity after five years of their treatment (Fig. S4 in ESM). In a third scenario, we combined these two assumptions. 
In PSA, we varied selected inputs simultaneously over 10,000 independent Monte Carlo trials using distributions that were assigned a priori (Table 1 and Table S3 in ESM). We summarized the results of the PSA in cost-effectiveness acceptability curves that plot the average probability that SoC and MDMA-AT have the highest NMV across a range of cost-effectiveness thresholds (\$0-100,000 per QALY gained).

\section{Results}

\subsection{Base-case}

Between 780,000 and 1.41 million patients with PTSD receive MDMA-AT under the three treatment targets (Fig. S5 in ESM). Achieving the 50\% target would avert an estimated 78,768 deaths and gain over 6 million discounted QALYs over 10 years (Table 2). Achieving the $25 \%$ target can save 43,618 lives and gain 3.3 million QALYs over 10 years. The $75 \%$ treatment target saves most lives $(106,932)$ and gains the most QALYs (8.2 million) over 10 years.

In all three targets, the costs of expanding access to MDMA-AT to eligible patients is less than the costs of providing SoC to the same number of patients. As a result, all three treatment targets are dominant (i.e., cost-saving while producing health benefits).

\subsection{One-way Sensitivity Analysis}

One-way sensitivity analysis found that using the highest and lowest values of various inputs in our model did not change the main finding that MDMA-AT is dominant when compared to SoC (Tables S4-S6 in ESM).
The influence of various inputs on the NMV of MDMA-AT are shown in Fig. S6-S8 in the ESM for the 25\%, 50\%, and 75\% treatment targets, respectively. The results show that across all three treatment targets, MDMA-AT has a positive NMV, even when the most extreme values of any input are used in the model, which means that the benefits of the intervention outweigh its costs. The most influential parameters for all three targets are the time horizon used (10-40 years) and the number of eligible patients with chronic and severe PTSD (2.11-4.74 million).

\subsection{Scenario Analysis}

The results of the scenario analyses are found in Table S7 in ESM. We found that reducing the effectiveness of MDMAAT or increasing the effectiveness of SoC does not change the main finding that MDMA-AT is dominant in all three treatment targets.

\subsection{Probabilistic Sensitivity Analysis}

Across 10,000 independent simulations, we found that MDMA-AT is dominant compared to SoC, and the average results (Table S8 in ESM) were similar to our base-case findings (Table 2). The results of the PSA are further summarized in cost-effectiveness planes (Fig. S9-S11 in ESM) and cost-effectiveness acceptability curves (Fig. S12-S14 in ESM). After accounting for multi-parameter uncertainty, MDMA-AT under any treatment target was found to be the optimal choice (i.e., provide greater NMV) $100 \%$ of the time across various cost-effectiveness thresholds.

\section{Discussion}

This study estimated the economic and public health benefits of MDMA-AT for treating chronic and severe PTSD. We found that expanding access to MDMA-AT to 25-75\%

Table 2 Summary of main results

\begin{tabular}{|c|c|c|c|c|c|c|}
\hline & \multicolumn{2}{|l|}{$25 \%$ target } & \multicolumn{2}{|l|}{$50 \%$ target } & \multicolumn{2}{|l|}{$75 \%$ target } \\
\hline & Standard of care & MDMA-AT & Standard of care & MDMA-AT & Standard of care & MDMA-AT \\
\hline Deaths & 191,199 & 147,581 & 347,618 & 268,850 & 475,175 & 368,243 \\
\hline QALYs & $6,491,139$ & $9,818,489$ & $11,723,620$ & $17,736,175$ & $15,917,517$ & $24,085,168$ \\
\hline Costs (billions US\$) & 256 & 147 & 462 & 266 & 628 & 361 \\
\hline Incremental deaths averted & 43,618 & & 78,768 & & 106,932 & \\
\hline Incremental QALYs & $3,327,350$ & & $6,012,555$ & & $8,167,652$ & \\
\hline $\begin{array}{l}\text { Incremental cost-savings } \\
\text { (US\$) }\end{array}$ & $\$ 109$ billion & & $\$ 196$ billion & & $\$ 266$ billion & \\
\hline
\end{tabular}

Incremental cost-effectiveness ratios were not calculated because MDMA-AT was lower in cost and produced more health benefits than standard of care for three MDMA-AT treatment targets. Costs are in billions US\$

$M D M A-A T$ methylenedioxymethamphetamine-assisted psychotherapy, $Q A L Y$ quality-adjusted life year 
of eligible patients over 10 years would avert between 43,618-106,932 deaths, produce 3.3-8.2 million discounted QALYs, and lead to \$109-\$266 billion in discounted savings for the healthcare system.

The findings from this study suggest that MDMA-AT is comparable to other widely used therapies in the USA. For example, the number of deaths averted by expanding access to MDMA-AT is higher than the estimated number of suicides prevented by the availability of fluoxetine for depression $(33,600$, credible interval 22,400-45,000) [39]. The number of QALYs gained through MDMA-AT is more than antiretroviral therapy during the first few years of the HIV/ AIDS epidemic (2.95 million) [40] or achieving national targets for HIV testing and treatment over 20 years (2.14 million) [41]. Finally, the costs savings we estimated in this study are comparable to the direct medical expenditures that can be averted by access to smoking cessation programs $[42,43]$.

Our study builds on two economic evaluations of MDMA-AT that demonstrated the value of this intervention. Using pooled results from six Phase 2 trials [19], one study estimated that MDMA-AT for 1000 patients with PTSD can generate $\$ 103.2$ million in net savings and 5553 QALYs over 30 years when compared to SoC [25]. Under the most conservative assumption that benefit ceases after 12 months, the ICER is $\$ 26,427$ per QALY gained, significantly lower than the $\$ 100,000$ per QALY gained threshold that is commonly used to evaluate the efficiency of health interventions in the USA [37]. A more recent study used data from the Phase 3 trial of MDMA-AT, which involved one additional MDMA session than in the Phase 2 trial, and found that providing 1000 patients with PTSD access to MDMA-AT would save the healthcare system approximate \$132.9 million while averting 61.4 premature deaths and generating 4856 QALYs over 30 years [24].

Our study also finds that MDMA-AT is cost-saving under a range of assumptions, although expanding access to MDMA-AT requires significant initial outlay. To realize the savings we estimated from this study, public and private payers, health systems, and government will have to invest in human resources to administer MDMA-AT. For example, the Phase 3 trial protocol involved 15 sessions with two trained therapists, which means that treating $25-75 \%$ of eligible patients with MDMA-AT (as we modeled in this study) will require training and certifying thousands of therapists.

As with any new therapy, other scientific, political, and legal factors will also shape the speed and scale in which MDMA-AT will be adopted [44]. Multidisciplinary Association for Psychedelic Studies (MAPS), the Phase 3 trial sponsor, obtained an FDA "breakthrough therapy" designation for MDMA-AT in 2017, which promises to expedite development and review processes [45]. Although not required by the FDA, MAPS is currently enrolling subjects for an additional Phase 3 trial and expects to apply for full FDA approval for MDMA-AT by 2023 [23].

Federal and state action is also needed to fully legalize the production and prescription of MDMA-AT. Congress or the Drug Enforcement Administration, for instance, may fully deschedule MDMA, as was done for cannabidiol in 2018, a non-psychoactive ingredient found in cannabis that is used to produce the FDA-approved seizure medication Epidolex $^{\circledR}$. Descheduling would exempt MDMA from controlled substance restrictions, enable the FDA to regulate it as an approved therapeutic, and provide access to federal research dollars [46, 47]. FDA approval and descheduling of MDMA is also a necessary condition for private and public payers to include MDMA-AT as a benefit; thus, without such approvals, the level of access explored in the current analysis would be impossible.

Aside from supply-side factors, demand-side determinants will have to be addressed to achieve the savings and health benefits we estimated. For example, our model assumes that there are no financial barriers to care, and all those who are eligible and wish to receive treatment can do so via sufficient insurance coverage or self-financing. Additionally, our study assumes high levels of care-seeking and treatment adherence among people with PTSD, which may not reflect reality given the stigma around severe mental illness and other cognitive, cultural, economic, and logistical barriers to PTSD care, which have been documented in the literature [48-50].

\subsection{Limitations}

This study has several limitations. First, there is uncertainty around the true number of eligible patients with chronic and severe PTSD for MDMA-AT. Estimates of the number of people with PTSD are limited and challenging to calculate because PTSD cases, like many mental illnesses that are highly stigmatized, are often underreported. There are also no precise estimates of the proportion of PTSD cases that are chronic and severe, and, among these cases, no studies to our knowledge have estimated the prevalence of comorbidities that would make patients ineligible for MDMA-AT. For this study, we used the prevalence of any current alcohol and substance use disorder, which is the most common comorbidity in PTSD patients, to determine the proportion of cases that would be ineligible for MDMA-AT. However, we used data from the Veterans Administration, and the prevalence from that patient population may be different from that of the broader public. Additional research is needed to refine this input.

Second, we modeled a closed cohort of patients with chronic and severe PTSD, and we have likely underestimated the benefits of access to MDMA-AT over the time 
horizon of our study. Future studies should include incident (in addition to prevalent) cases of PTSD, although estimates of the incidence of PTSD are limited and often focus on specific groups of people that experience a traumatic event, such as a natural disaster or acute violent episodes [51-53]. Third, we used a healthcare payer perspective, and future analyses should use a societal perspective that include other sources of costs and benefits, such as patient time costs, travel costs, caregiver burden, and lost productivity and consumption [26].

Fourth, our model relies primarily on the inputs and findings of a previous cost-effectiveness analysis, which means that limitations from that study carry over to our current study. For example, Marseille et al assumed that patients with PTSD who receive SoC experience no improvements in their disease severity. This assumption is supported by evidence that chronic and severe PTSD is highly treatment-resistant, and that spontaneous remission in PTSD is limited to the first years following diagnosis $[17,22,35,54]$. In addition, we assumed that the efficacy of MDMA-AT is durable, which is supported by limited research showing that patients who have received MDMA-AT maintain statistically and clinically significant improvements in their severe PTSD symptoms for years following treatment [35]; however, the true long-term efficacy of MDMA-AT remains unknown. We conducted scenario analyses to relax these assumptions, and future studies should further explore the effect of this assumption on the efficiency of MDMA-AT.

Finally, the generalizability of our results is limited by the underlying Phase 3 trial which (1) only evaluated the shortterm efficacy of MDMA-AT and (2) involved participants who do not necessarily represent the diversity of the US patients with chronic and severe PTSD.

\section{Conclusion}

MDMA-AT has the potential to significantly improve PTSD care nationwide. Increased access to this therapy can save and extend lives of patients with chronic and severe PTSD, as well as reduce healthcare costs.

Supplementary Information The online version contains supplementary material available at https://doi.org/10.1007/s40261-022-01122-0.

\section{Declarations}

Funding No funding was received to conduct this study.

Conflicts of interest All authors have no conflict of interest to declare.

Ethics approval Not applicable.
Consent to publish Not applicable.

Consent to participate Not applicable.

Availability of data and materials Data needed to replicate this study are found in the main text, cited references, and in the electronic supplementary material.

Code availability Not applicable.

Author contributions ALVA validated, curated, analyzed, and interpreted the data, wrote the first draft of the manuscript, edited the manuscript, and is the guarantor of the study. JGK conceptualized the study, edited the manuscript, and provided oversight. EM conceptualized the study, curated, analyzed, and interpreted the data, edited the manuscript, and provided oversight and supervision. All authors approve the submission and publication of the manuscript.

Open Access This article is licensed under a Creative Commons Attribution-NonCommercial 4.0 International License, which permits any non-commercial use, sharing, adaptation, distribution and reproduction in any medium or format, as long as you give appropriate credit to the original author(s) and the source, provide a link to the Creative Commons licence, and indicate if changes were made. The images or other third party material in this article are included in the article's Creative Commons licence, unless indicated otherwise in a credit line to the material. If material is not included in the article's Creative Commons licence and your intended use is not permitted by statutory regulation or exceeds the permitted use, you will need to obtain permission directly from the copyright holder. To view a copy of this licence, visit http://creativecommons.org/licenses/by-nc/4.0/.

\section{References}

1. Kilpatrick DG, Resnick HS, Milanak ME, Miller MW, Keyes KM, Friedman MJ. National estimates of exposure to traumatic events and PTSD prevalence using DSM-IV and DSM-5 criteria. J Trauma Stress. 2013;26:537-47.

2. Goldstein RB, Smith SM, Chou SP, Saha TD, Jung J, Zhang H, et al. The epidemiology of DSM-5 posttraumatic stress disorder in the United States: results from the National Epidemiologic Survey on Alcohol and Related Conditions-III. Soc Psychiatry Psychiatr Epidemiol. 2016;51:1137-48.

3. Kessler RC, Chiu WT, Demler O, Walters EE. Prevalence, severity, and comorbidity of 12-month DSM-IV disorders in the National Comorbidity Survey Replication. Arch Gen Psychiatry. 2005;62:617-27.

4. Nichter B, Norman SB, Maguen S, Pietrzak RH. Moral injury and suicidal behavior among US combat veterans: Results from the 2019-2020 National Health and Resilience in Veterans Study. Depress Anxiety. 2021;38:606-14.

5. Steenkamp MM, Litz BT, Hoge CW, Marmar CR. Psychotherapy for military-related PTSD: a review of randomized clinical trials. JAMA. 2015;314:489-500.

6. Seal KH, Bertenthal D, Miner CR, Sen S, Marmar C. Bringing the war back home: mental health disorders among 103788 US veterans returning from Iraq and Afghanistan seen at Department of Veterans Affairs facilities. Arch Intern Med. 2007;167:476.

7. Dursa EK, Reinhard MJ, Barth SK, Schneiderman AI. Prevalence of a Positive Screen for PTSD Among OEF/OIF and OEF/OIF-Era Veterans in a Large Population-Based Cohort. J Trauma Stress. 2014;27:542-9. 
8. Helzer JE, Robins LN, McEvoy L. Post-traumatic stress disorder in the general population: findings of the Epidemiologic Catchment Area Survey. N Engl J Med. 1987;317:1630-4.

9. von der Warth R, Dams J, Grochtdreis T, König H-H. Economic evaluations and cost analyses in posttraumatic stress disorder: a systematic review. Eur J Psychotraumatology. 2020;11:1753940.

10. Haviland MG, Banta JE, Sonne JL, Przekop P. Posttraumatic stress disorder-related hospitalizations in the United States (20022011): rates, co-occurring illnesses, suicidal ideation/self-harm, and hospital charges. J Nerv Ment Dis. 2016;204:78-86.

11. Kartha A, Brower V, Saitz R, Samet JH, Keane TM, Liebschutz J. The impact of trauma exposure and post-traumatic stress disorder on healthcare utilization among primary care patients. Med Care. 2008;46:388-93

12. Vyas KJ, Fesperman SF, Nebeker BJ, Gerard SK, Boyd ND, Delaney EM, et al. Preventing PTSD and depression and reducing healthcare costs in the military: a call for building resilience among service members. Mil Med. 2016;181:1240-7.

13. Tuerk PW, Wangelin B, Rauch SAM, Dismuke CE, Yoder M, Myrick H, et al. Health service utilization before and after evidence-based treatment for PTSD. Psychol Serv. 2013;10:401-9.

14. Outcalt SD, Yu Z, Hoen HM, Pennington TM, Krebs EE. Health care utilization among veterans with pain and posttraumatic stress symptoms. Pain Med. 2014;15:1872-9.

15. Kaier E, Possemato K, Lantinga LJ, Maisto SA, Ouimette P. Associations between PTSD and healthcare utilization among OEF/OIF veterans with hazardous alcohol use. Traumatology. 2014;20:142-9.

16. Steinert C, Hofmann M, Leichsenring F, Kruse J. The course of PTSD in naturalistic long-term studies: high variability of outcomes. A systematic review. Nord J Psychiatry. 2015;69:483-96.

17. Morina N, Wicherts JM, Lobbrecht J, Priebe S. Remission from post-traumatic stress disorder in adults: a systematic review and meta-analysis of long term outcome studies. Clin Psychol Rev. 2014;34:249-55.

18. Koek RJ, Schwartz HN, Scully S, Langevin J-P, Spangler S, Korotinsky A, et al. Treatment-refractory posttraumatic stress disorder (TRPTSD): a review and framework for the future. Prog Neuropsychopharmacol Biol Psychiatry. 2016;70:170-218.

19. Mithoefer MC, Feduccia AA, Jerome L, Mithoefer A, Wagner M, Walsh Z, et al. MDMA-assisted psychotherapy for treatment of PTSD: study design and rationale for Phase 3 trials based on pooled analysis of six Phase 2 randomized controlled trials. Psychopharmacology. 2019;236:2735-45.

20. Institute of Medicine. Treatment for posttraumatic stress disorder in military and veteran populations: final assessment. Washington, D.C.: National Academies Press; 2014.

21. Bradley R, Greene J, Russ E, Dutra L. A multidimensional meta-analysis of psychotherapy for PTSD. Am J Psychiatry. 2005;162:214-27.

22. Mitchell JM, Bogenschutz M, Lilienstein A, Harrison C, Kleiman S, Parker-Guilbert K, et al. MDMA-assisted therapy for severe PTSD: a randomized, double-blind, placebo-controlled Phase 3 study. Nat Med. 2021;27:1025-33.

23. Multidisciplinary Association for Psychedelic Studies. MDMAassisted psychotherapy. 2021. https://maps.org/research/mdma. Accessed 9 Jul 2021.

24. Marseille E, Mitchell JM, Kahn JG. Updated cost-effectiveness of MDMA-assisted therapy for the treatment of PTSD: findings from a Phase 3 trial. PLoS One. 2022 (In press).

25. Marseille E, Kahn JG, Yazar-Klosinski B, Doblin R. The cost-effectiveness of MDMA-assisted psychotherapy for the treatment of chronic, treatment-resistant PTSD. PLoS ONE. 2020;15:e0239997.
26. Neumann PJ, Sanders GD, Russell LB, Siegel JE, Ganiats TG, editors. Cost-effectiveness in health and medicine. 2nd ed. New York: Oxford University Press; 2017.

27. Husereau D, Drummond M, Petrou S, Carswell C, Moher D, Greenberg D, et al. Consolidated Health Economic Evaluation Reporting Standards (CHEERS) statement. BMJ. 2013;346:f1049-f1049.

28. Office of Disease Prevention and Health Promotion. Increase the proportion of adults with serious mental illness who get treatment. Healthy People 2030. 2021. https://health.gov/healthypeo ple/objectives-and-data/browse-objectives/mental-health-andmental-disorders/increase-proportion-adults-serious-mental-illne ss-who-get-treatment-mhmd-04. Accessed 9 Jul 2021.

29. Chisholm D, Sweeny K, Sheehan P, Rasmussen B, Smit F, Cuijpers $P$, et al. Scaling-up treatment of depression and anxiety: a global return on investment analysis. Lancet Psychiatry. 2016;3:415-24.

30. Chisholm D, Heslin M, Docrat S, Nanda S, Shidhaye R, Upadhaya $\mathrm{N}$, et al. Scaling-up services for psychosis, depression and epilepsy in sub-Saharan Africa and South Asia: development and application of a mental health systems planning tool (OneHealth). Epidemiol Psychiatr Sci. 2017;26:234-44.

31. National Institute of Mental Health. Post-traumatic stress disorder (PTSD). 2017. https://www.nimh.nih.gov/health/statistics/posttraumatic-stress-disorder-ptsd. Accessed 9 Jul 2021.

32. U.S. Census Bureau. Age and sex composition in the United States: 2019. https://www.census.gov/data/tables/2019/demo/ age-and-sex/2019-age-sex-composition.html. Accessed $9 \mathrm{Jul}$ 2021.

33. Hefner K, Rosenheck R. Multimorbidity among veterans diagnosed with PTSD in the Veterans Health Administration nationally. Psychiatr Q. 2019;90:275-91.

34. MAPS Public Benefit Corporation. Protocol MAPP1. 2020. http://maps.org/mapp1. Accessed 9 Jul 2021.

35. Mithoefer MC, Wagner M, Mithoefer A, Jerome L, Martin S, Yazar-Klosinski B, et al. Durability of improvement in posttraumatic stress disorder symptoms and absence of harmful effects or drug dependency after 3,4-methylenedioxymethamphetamine-assisted psychotherapy: a prospective long-term follow-up study. J Psychopharmacol. 2013;27:28-39.

36. Walker EA, Katon W, Russo J, Ciechanowski P, Newman E, Wagner AW. Health care costs associated with posttraumatic stress disorder symptoms in women. Arch Gen Psychiatry. 2003;60:369.

37. Neumann PJ, Cohen JT. QALYs in 2018-advantages and concerns. JAMA. 2018;319:2473.

38. Pickard AS, Law EH, Jiang R, Pullenayegum E, Shaw JW, Xie F, et al. United States valuation of EQ-5D-5L health states using an international protocol. Value Health. 2019;22:931-41.

39. Milane MS, Suchard MA, Wong M-L, Licinio J. Modeling of the temporal patterns of fluoxetine prescriptions and suicide rates in the United States. PLoS Med. 2006;3:e190.

40. Walensky RP, Paltiel AD, Losina E, Mercincavage LM, Schackman BR, Sax PE, et al. The survival benefits of AIDS treatment in the United States. J Infect Dis. 2006;194:11-9.

41. Borre ED, Hyle EP, Paltiel AD, Neilan AM, Sax PE, Freedberg KA, et al. The clinical and economic impact of attaining National HIV/AIDS Strategy Treatment targets in the United States. J Infect Dis. 2017;216:798-807.

42. American Lung Association. Smoking cessation-the economic benefits. 2020. https://www.lung.org/policy-advocacy/tobacco/ cessation/smoking-cessation-economic-benefits. Accessed $9 \mathrm{Jul}$ 2021.

43. Baker CL, Ding Y, Ferrufino CP, Kowal S, Tan J, Subedi P. A cost-benefit analysis of smoking cessation prescription 
coverage from a US payer perspective. Clin Outcomes Res CEOR. 2018;10:359-70.

44. Jordans MJD, Kohrt BA. Scaling up mental healthcare and psychosocial support in low-resource settings: a roadmap to impact. Epidemiol Psychiatr Sci. 2020;29:e189.

45. Feduccia AA, Jerome L, Yazar-Klosinski B, Emerson A, Mithoefer MC, Doblin R. Breakthrough for trauma treatment: safety and efficacy of MDMA-assisted psychotherapy compared to paroxetine and sertraline. Front Psychiatry. 2019;10:650.

46. Congressional Research Service. State marijuana "legalization" and federal drug law: a brief overview for Congress. https://fas. org/sgp/crs/misc/LSB10482.pdf. Accessed 9 Jul 2021.

47. Haffajee R, Mauri A. Cannabis liberalization in the US: the policy landscape. Health Aff. 2021. https://www.healthaffairs.org/do/10. 1377/hpb20210518.36548/full/. Accessed 25 Feb 2022.

48. Kazlauskas E. Challenges for providing healthcare in traumatized populations: barriers for PTSD treatments and the need for new developments. Glob Health Action. 2017;10:1322399.

49. Sayer N, Friedemann-Sánchez G, Spoont M, Murdoch M, Parker L, Chiros C, et al. A qualitative study of determinants of PTSD treatment initiation. Psychiatry. 2009;72:238-55.

50. Lord S, Wagner A, Russo J, Love J, Zatzick D. Assessing barriers to care and readiness for cognitive behavioral therapy in early acute care PTSD interventions. Psychiatry. 2011;74:207-23.

51. Yule W, Bolton D, Udwin O, Boyle S, O'Ryan D, Nurrish J. The long-term psychological effects of a disaster experienced in adolescence: I: the incidence and course of PTSD. J Child Psychol Psychiatry. 2000;41:503-11.

52. Caldwell MF. Incidence of PTSD among staff victims of patient violence. Hosp Community Psychiatry. 1992;43:838-9.
53. de Bocanegra HT, Moskalenko S, Kramer EJ. PTSD, depression, prescription drug use, and healthcare utilization of Chinese workers affected by the WTC attacks. J Immigr Minor Health. 2006;8:203-10.

54. Solomon Z, Mikulincer M. Trajectories of PTSD: a 20-year longitudinal study. Am J Psychiatry. 2006;163:659-66.

55. FAIR Health Consumer. Welcome to FAIR Health. 2021. http:// www.fairhealthconsumer.org. Accessed 9 Jul 2021.

56. Centers for Medicare \& Medicaid Services. Overview of the Medicare physician fee schedule search. 2021. https://www.cms. gov/medicare/physician-fee-schedule/search/overview. Accessed 9 Jul 2021.

57. Ivanova JI, Birnbaum HG, Chen L, Duhig AM, Dayoub EJ, Kantor ES, et al. Cost of post-traumatic stress disorder vs major depressive disorder among patients covered by Medicaid or private insurance. Am J Manag Care. 2011;17:10.

58. Chan D, Cheadle AD, Reiber G, Unützer J, Chaney EF. Health care utilization and its costs for depressed veterans with and without comorbid PTSD symptoms. Psychiatr Serv. 2009;60:1612-7.

59. Lavelle TA, Kommareddi M, Jaycox LH, Belsher B, Freed MC, Engel CC. Cost-effectiveness of collaborative care for depression and PTSD in military personnel. Am J Manag Care. 2018;24:91-8.

60. Marciniak MD, Lage MJ, Dunayevich E, Russell JM, Bowman L, Landbloom RP, et al. The cost of treating anxiety: the medical and demographic correlates that impact total medical costs. Depress Anxiety. 2005;21:178-84.

61. Arias E, Xu J. United States life tables, 2018. Natl Vital Stat Rep. 2020;69:1-44. 